\section{Ant Addregs}

\author{
ON THE
}

\section{DIAGNOSIS AND TREATMENT OF ACUTE INFLAMMATIONS OF THE UPPER ABD0NEN.}

Delivered before the Norfolk aNd Norwich MedicoChirurgical Society, October 12th, 1909,

\section{By A. W. MAYO ROBSON, D.Sc., F.R.C.S.}

AcUTE peritonitis of the upper abdomen may be dependent on disease starting in the stomach, duodenum, jejunum, colon, gall bladder, bile ducts, pancreas, liver, spleen, upward displaced appendix, kidneys, or inflamed lymphatic glands, and may be due to ulcer, perforation, abscess, concretions, hydatids, cancer, internal hernia, or other pathological conditions.

The acute seizure may occur after well-marked sym ptoms of disease extending over long periods, or may come on suddenly without premonitory symptoms to indicate the cause; in the former, the diagnosis is, as a rule more simple, but in the latter it may be most difficult, and may sometimes be even impossible without exploration.

In nearly every case pain is the prominent symptom and it is usually associated with rigidity of one or both recti and with local and general tenderness.

In other cases profound collapse may be the first sign, and the site of the trouble may only be indicated by rigidit of the muscles, by tenderness, and at times, in case of perforation of a hollow viscus, by absence of liver dullneas and a thrill obtained on delicate palpation.

In the earlier stages the temperature is no guide, though htar it may afford valuable information.

Pinched features, an anxious expression of countenance, and general distress are usually seen in the acute cases, but are less marked in conditions coming on gradually.

Shallow breathing, immobility of the lower ribs and of the abdominal muscles are characteristic of peritonitis in the upper abdomen from whatever cause.

\section{High Appendicitis.}

I think it is not sufficiently recognized that acute appendicitis nearly always commences by sudden pain above the umbilicus, and only after some hours does the pain settle in the caecal region, though from the first rigidity of the lower part of the right rectus and tender ness at some point between the umbilicus and right anterior superior spine of the ilium will usually indicate the true nature of the disease.

If, however, the caecum be displaced upwards, or the appendix be unusually long and extend upwards, appendicitis may lead to confusion in diagnosis, as in the following case :

A gentleman aged 27, seen in June, 1907, had had several attacks within the year of pain starting just below and to th right of the ensiform cartilage, associated with rigidity of the upper part of the right rectus, and tenderness at a point between the ninth costal cartilage and the umbilicus, but nearer to the former. Each attack was associated with vomiting and some general abdominal distension, and was followed by sallowness, but without definite jenndice. After a severe seizure the abdomen was opened by a vertical incision below the right costal margin when an inflamed appendix nearly seven inches costal margin, when an inflamed appendix, nearly seven inche in length, was found containing pus, the tip being adherent to the liver, though the caecum was in its nsual place. The bil passages and the duodenum were involved in adhesions, but otherwise healthy,

In lete recovery.

In another case a displaced appendix had been slightly inflamed on a number of occasions during several year antecedent to the attack in question, and the inflammation presented the ordinary signs of catarrhal appendicitis. When seen by $m e$ in consultation in February, 1906, the patient had had rigors for three days. His breath was very foul, he looked extremely ill, and was evidently suffering from septicaemia. In this case all the tendernes was above the umbilicus under the right costal margin and the caecal region could be pressed on without pain Although there wag no ceneral abdomingl distension signs of Althe fluid conld be licito the previous history of dite a symptoms and signs then present, a diagnosis of appendicitis 4 the abdomen by a vertical incision starting from the right costal margin, the colon, caecum, and gall bladder were found adherent, and behind them was a gangrenous perforated appendix surrounded by offensive pus; the gall bladder also inflamed, and cols aso inflamed, and contained brownish offensive matter and several gall stones. All free fluid was wiped away, the appendix removed, and the gall bladder emptied and drained. The local symptoms were relieved and recovery looked likely,
but the pyaemic symptoms continued and ultimately proved fatal.

'The patient's extreme illness decided the question of draining instead of removing the gall bladder; but I now think cholecystectomy ought to have been performed, as in another similar case I removed both the gall bladder and appendix, both being acutely inflamed, with complete success.

Acute inflammation and abscess in the upper abdomen secondary to appendix trouble is well known, and I could give several examples in which obscure symptoms have followed on appendicitis, and have been made clear by the discovery of a subphrenic abscess that had to be treated by incision and drainage below or between the ribs.

The history of an attack of appendicitis presenting the usual signs, followed after some days or even two or three weeks by fever, rigors, and pain behind the right costal margin, and associated with rigidity of the right rectus, should give rise to the suspicion of this complication and lead to exploration.

In one case that I operated on last year in Italy, in a second week of diagnosed appendicitis, there was marked tenderness beneath the right ribs, with rigid right rectus, and, after removing a perforated appendix, I traced the course of an abscess behind the whole length of the ascending colon up to the hepatic region, and thus apparently saved the patient from what would have been a subphrenic abscess.

In another case, some weeks after apparent recovery from appendicitis, fever, with rigors and rapid pulse associated with swelling, pain, and tenderness in the region of the liver, led to the diagnosis of secondary subphrenic abscess, which was treated by incision below the right costal arch and intercostal drainage.

In some of these cases, according to the general condition of the patient, the incision should be extended downwards, and at the same time the primary trouble in the appendix removed, while in others the appendix may be left for a later operation.

Where a suspicion of appendix trouble arises in a case in which the focus of acute inflammation renders it desirable to open the abdomen abore the umbilicus, I am accustomed to make my incision about an inch to the right of the mid-line, and to separate and draw outwards the rectus, thus enabling the abdominal wall to be efficiently repaired by suturing the anterior and posterior rectus sheaths separately. In this way the incision may be safely extended downward as far as necessary, with the knowledge that no weakness will be left.

\section{Acute Cholecystitis.}

This is one of the commonest causes of inflammation in the upper abdomen. It may be secondary to cholelithiasis or to typhoid fever, or may come on without any apparent primary cause.

In some of these cases the diagnosis is readily made from the previous history of gall-stone attacks, as well as from a swelling of the gall bladder and right lobe of the liver associated with local tenderness; in others, the local symptoms and signs are so definite that, although there may be no previous history pointing to gall-bladder trouble, the diagnosis is not difficult; but in some cases an exact diagnosis may be almost impossible, as the symptoms may be so acute as to simulate perforation of the stomach or duodenum, or some other acute abdominal catastrophe. For instance, I operated on an elderly lady some little time ago on the secoud day of an acute phlegmonous cholecystitis, and there had never been any symptoms of cholelithiasis to give a clue to the cause of the trouble; and only about three months ago I operated on a lady in Dorsetshire and removed a gangrenous gall bladder within forty-eight hours of the onset of the attack, but, although an accurate diagnosis had been made before my arrival, chiefly by the local signs, the history of indigestion, pointing to duodenum or pylorus, made it quite 
possible that a perforated ulcer might be the cause of the peritonitis then present.

In obtaining the history, it should not be forgotten that attacks of pain so long ago as twenty, thirty, or forty years may have significance, nor must it be forgotten that an enlargement of the right lobe of the liver very often accom. panies cholelithiasis, and may completely hide the inflamed gall bladder, though the tender spot between the ninth costal cartilage and the umbilicus will be seldom absent. Absence of janndice has no diagnostic value, as the obstrue. tion is usually in the cystic duct; but in many of these cases inflammation extending to the common duct or to the head of the pancreas sets up secondary jaundice, which has then positive diagnostic importance.

Did time allow, I could relate numerous examples of

(a) Acute cholecystitis and gangrene of the gall bladder associated with gall stones, but without any previous symptoms pointing to the same; of

(l) Acute cholecystitis due to cholelithiasis, with previous though long antecedent history of gall stones; and of

c) Acute cholecystitis without gall stones, and without any previous history pointing to trouble in the gall bladder region.

The treatment of these cases is by cholecystectomy, for the gall bladder is so seriously damaged that, if left, it may not only prove immediately dangerous, but may give rise to ulterior trouble.

Under ordinary circumstances, in operating for gall stones, I am not in favour of removing the gall bladder, as I do not think it to be the useless organ that some surgeons describe it to be.

Seeing that recurrence of gall stones after cholecystotomy with efficient drainage is an extremely rare event, that the danger of cholecystotomy in uncomplicated cholelithiasis is almost nil-in my own experience not 1 per cent. over a large number of cases-that drainage of the bile passages after any gall-stone operation is distinctly advantageous, and that if at some future time it should be necessary to drain the bile passages-say for obstructive jaundice or interstitial pancreatitis - the difficulties would be increased by the absence of a gall bladder, though not by a cholecystotomy, my feeling is very decided that cholecystectomy should not replace the simple operation of cholecystotomy as a routine procedure in operating for gall stones, though it is indicated in phlegmonous choleeystitis, and usually in empyema of the gall bladder, and in other conditions not coming under review in my present paper.

But in connexion with the gall bladder and bile ducts, a condition equally, if not more, serious may be found leading to acute peritonitis in the upper abdomen, and that is ulceration and perforation leading to extravasation of the infective contents of the biliary passages.

The indications for treatment in such serious conditions are important, as not infrequently the operator is tempted to do too much. If the case be seen sufficiently early, before the system is poisoned and before the peritonitis has become diffused, a complete operation, with removal of the ulcerated and perforated gall bladder and free drainage of the right kidney pouch, will be called for; but if diffusion has occurred and general peritonitis is present, an exposure of the inflammatory focus, a rapid removal of the pus and inflammatory material in the gall bladder region, and free drainage of the right kidney pouch through the loin, and, if needful, of the pelvic pouch through a suprapubic puncture, are only called for, and any attempt at abdominal flushing, removal of the gall bladder, or in fact any serious prolongation of the operation, will probably end in disaster. The following cases afford illustrations:

I operated in 1893 on a case of ruptured gall bladder, due to gall stones, in which acute symptoms supervened suddenly after a history of over twenty years' gall-stone seizures. On the third day of the attack, the patient being extremely ill with all the signs of acute peritonitis in the right upper abdomen, all the signs of acute peritonitis in the right upper abdomen, I made a free incision below the right costat margin and ill to bear a prolonged search, I simply inserted drainage tubes into the subphrenic and right renal regions, with the result that the patient not only recovered, but was well some years later without any further operative treatment having been necessary.

Three years ago, in a patient nearly 70 years of age, I operated in like manner, the patient being at the time in an extremely critical condition on account of pneumonia of the right base, albuminuria, and a feeble heart. The sudden acute attack,
followed by peritonitis of the right upper abdomen with fixation of the ribs, rigid right rectus, and a doubtful sense of fluctuation, left no doubt as to the nature of the disease, an'd on opening the abdomen above and to the right of the umbilicus. a large quantity of offensive pus and bile with a number of gall stones were evacuated from under the diaphragm and beneath the liver. Simple drainage by large tubes, one of which was passed between the ninth and tenth ribs, and one through a stah wound in the loin, led to complete recovery, although no attempt was made to close the perforation or to remove the gall bladder. The patient is at the present time in good health, and, curiously, has lost his albuminuria that had been present off and on for several years.

The condition is so grave that no hard-and-fast line of treatment can be insisted on. In the two cases just related a mere wiping out of the septic cavity after the removal of its contents, followed by free drainage through the loin in one case and between the ribs in the other, sufficed to bring about recovery, whereas a more extensive and radical operation would probably have resulted in disaster.

On the other hand, if the extravasation is local and the perforated gall bladder or cystic duct be ulcerated or gangrenous, and the patient in a fit condition to bear it, then removal will be the correct treatment, as in the following case :

I operated in May, 1906, on a gentleman of 62, during an acute gall-stone attack associated with signs of acute local peritonitis limited to the upper abdomen. On exposing the inflamed gall bladder, a collection of grumous, offensive pus was found beneath the liver. A perforation of the under surface of the gall bladder the liver. A perforation of the under surface of the gall bladder had allowed the contents, including gall stones, to escape, the the extravasated fluid and the concretions, the gall bladder was rapidly cut away by means of scissors, there being no haemorrapidly cut away by means of scissors, there being no haemor-
rhage on account of thrombosis of vessels. A tube was passed rhage on account of thrombosis of vessels. A tube was passed into the cystic duct, and the right renal pouch was drained
through a stab puncture in the loin. The patient is now in excellent health, though his recovery was somewhat tardy.

In case of extravasation from injury the conditions are quite different, as the effused fluids are not septic ; therefore, if the condition of ruptured gall bladder or bile ducts be recognized sufficiently early, a complete operation may be safely performed, and the gall bladder can be removed or drained or a ruptured bile duct may be sutured or drained. The right kidney pouch should also be drained through a lumbar stab puncture. In these cases, besides the local signs of peritonitis, jaundice supervenes about the third day, owing to absorption of some of the effused bile.

Should the serious nature of the accident not be recognized until several days have elapsed and a localized collection form beneath the right costal margin, it will be rarely advisable to do more than perform simple incision and drainage. Should the extravasated fluids have overflowed into the general cavity of the peritoneum the local drain may be supplemented by suprapubic drainage, assisted by the patient being well propped up in bed so as to facilitate the evacuation of the extravasated fluid through the tabe.

If adhesions have taken place, ulceration may occur into neighbouring organs, setting up symptoms of local peritonitis associated with those pointing to intestinal obstruction, in which case a complete operation may have to be undertaken, as in the following case:

A gentleman of 76, whom I saw on the third day of an acute attack of pain in the upper abdomen and violent vomiting, had never had any symptoms pointing to gall stones, and had been quite well before the present seizure. The upper abdomen was distended and tender and the patient extremely ill. On opening the abdomen, acute cholecystitis with ulceration, extending into the stomach and colon, was found, and a large gall stone was removed from the interior of the stomach and one from the opening leading into the colon. After packing off the abdomina cavity by sterilized gauze and separating adhesions, a quantity of pus and bile was mopped up, the visceral openings closed,
and the ruptured gall bladder removed. The operation, which occupied half an hour, was completed with the patient in good condition, and when heard of a year later he was in good health.

\section{Perforated Duodenal Ulcer.}

This, while one of the most serious of the conditions we are considering, may occur without any warning and without any previous symptoms pointing even to the existence of ulcer.

I saw with his medical attendant a young man, aged 2l, within eight hours of a sudden attack of pain in the upper abdomen. the diagnosis having been made on the ground of the sudden 
onset of an agonizing pain beneath the right costal margin immediately after exertion, which was followed by shock, and associated with marked rigidity of the upper part of the right rectus, a fluid thrill felt on flicking the right superior abdominal rectus, a fluid thrill felt on flicking the right superior abdominal region, the presence of a tender spot to the right of and just above the umbilicus, and a history of a severe

chest a few weeks previous to the sudden illness.

There was an entire absence of digestive trouble preceding the attack. I immediately operated, and found a duodenal perforation the size of a threepenny piece, which I was able to suture and cover with a flap of omentum. As perforation had occurred on an empty stomach the extravasated fluid was comparatively small in amount and limited to the right side of the abdomen. Recovery was uninterrupted.

Or the condition may have been preceded by the symptoms of duodenal ulcer for years and yet occur at a time when least expected and when the patient is apparently quite well.

Out of a number of such cases I will select two examples.

One, a clergyman of middle age, on whom I operated fifteen years ago within twelve hours of the sudden onset of pain in the right upper abdomen, apparently caused by getting over a stile. He had had pain three to four hours after meals, relieved by taking food, off and on for several years, and had been awakened frequently about midnight, a very characteristic symptom of duodenal ulcer. In this case I recognized the perforation a once, and after closing it simply wiped out the effused fluid without drainage. The patient was well when heard of some years later.

The other example was in the case of a middle-aged medical man, whom I saw three days after perforation, suffering from general peritonitis. The attack occurred when he was getting into his dog-cart on his usual morning round of visits, and when I saw him he did not give the history of indigestion though later he recalled to mind that for a long time he had though later he recalled to mind that for a long

In this case the perforation was a large opening in the front part of the duodenum, which I closed, afterwards draining the right kidney pouch through a stab wound, and the pelvis through a suprapubic opening. Drainage was assisted by the semi-recumbent posture, and a good recovery resulted.

Perforated duodenal ulcer has often been mistaken for acute appendicitis, though the previous symptoms are usually so distinctive that the mistake ought not to occur so frequently.

If the case be seen within a few hours of the onset the mistake in diagnosis is of less importance, as, though an incision may be made in the appendix region, the nature of the extravasated fluid or the presence of a normal appendix will immediately throw light on the condition, and the incision can either be extended upwards or another incision made beneath the right costal margin for repair of the perforation.

But if at a later stage, when general peritonitis has developed, the mistake be made, and an appendix, inflamed secondarily as the result of a general peritonitis, be removed under the idea that it has originated the disease and the duodenal perforation be left, the patient wil almost certainly die, even though the peritoneal cavity be thoroughly drained.

Though general peritonitis from acute appendicitis is often followed by recovery after simple drainage, in peritonitis from duodenal ulcer the ulcer must be closed at the time of operation, as recovery has hardly, if ever, occurred where the perforation has been left untreated.

More than twenty years ago, having had success in the simple drainage of general peritonitis from perforated appendix, I was tempted to simply drain without closing a perforated duodenal ulcer on the third day after perforation, when the patient was, as I then thought, too feeble to bear an extension of the operation for the closing of the perforation; but, although the man survived till the third day, it was impossible to feed him, and the duodenal fistula continued to pour out fluid into the peritoneum.

The question as to the performance of gastro-enterostomy at the same time as the repair of a perforated gastric or duodenal ulcer is an interesting problem.

Though doubtless, for many reasons, gastro-enterostomy may be desirable, especially because it takes off strain from the newly-sutured opening, and at the same time enables the patient to be fed forthwith, yet such an extension of the operation is seldom possible in patients so ill as these subjects of perforation are, and I should only perform it at the time of closing a perforation in the duodenum where almost complete stenosis of the pylorus renders it certain that adequate feeding of the patient cannot be conducted subsequent to operation.

In several cases in which I have closed perforated duodenal ulcers in the presence of great thickening of the pylorus and first part of the duodenum the patients have recovered, and I have subsequently performed a successful gastro-enterostomy when conditions were more favourable; moreover, in some of these cases the patients have, mach to my astonishment, got completely well, and have not required a subsequent operation, although the pylorns appeared at the time to have a very small lumen.

The diagnosis of perforated duodenal ulcer rests on the previous history, the site and sudden character of the pain to the right of the umbilicus radiating to the loin, rigidity of the upper part of the right rectus, and the presence of fluid in the right of the abdomen. In perforated ulcer of the stomach there is usually a characteristic history of indigestion, with pain shortly after food, the pain being usually above and often to the left of the umbilicus, both recti being rigid and boardlike, and a fluid thrill being sometimes falt across the front of the abdomen.

Suppuration in connexion with chronic ulcer of the stomach or duodenum is often a sequence of subacute perforation in which a leak has occurred through a minute puncture in an empty viscus, giving time for adhesions to form between neighbouring organs, and so preventing a general peritonitis, but an abscess may also form by the spread of infection through the damaged stomach or duodenal wall apart from perforation.

The diagnosis of these cases presents fewer difficulties, as the history of gastric or duodenal ulcer over months or years preceding the attack in question is usually so distinct as to render evident the true nature of the disease, as in the following case:

A gentleman of leisure, aged 45, had suffered from acidity and flatulency off and on for years and from three to four hour after meals, moreover he was regularly awakened at 2 a.m. when the attacks were on, and had to take food to obtain relief clearly pointing to duodenal ulcer. When I saw him he was in grest distress with signs of inflammation beneath the right costal margin the right rectus muscle being rigid and the upper abdomen being extremely tender to the touch ; these acute. symptoms had come on suddenly three days previously, when the stomach was empty, and had increased in violence. A vertical incision below the right ribs revealed an abscess between the duodenum and liver, and at the bottom of the abscess was a minute perforation, just beyond the pyloras. The abscess cavity was carefully cleansed and the perforation closed.

Subsequen
results.

Some years ago I was asked to see a woman of 30 with a well marked left subphrenic abscess, the acute illness having been present for a fortnight, following on a sudden violent pain in the left upper abdomen. The history of pain immediately after food for many months left no doubt as to the cause being a chronic gastric ulcer, and the physical signs of a collection of fluid and gas under the left ribs showed that the abscess communicated with a hollow viscus. In this case drainage of the subphrenic abscess through an opening between the ninth and tenth ribs on the left side led to complete recovery.

But that the diagnosis is not always so easy the following case will show :

I was asked by a medical friend to see a gentleman, aged 53 who was supposed to be dying from a malignant tumour in the region of theliver, with secondary effusion into the right pleura and a hopeiess prognosis had been given. I elicited the history and a hopeiess prognosis had been given. I elicited the history that twenty years previously he had had a severe attack of indigestion, with pain some hours after food, and at that time he remembered that the illness was accompanied by dark motions, which his medical attendant told him were due to altered blood. From that time onwards he had been subject to
what he termed weak digestion, characterized by pain three to four hours after a meal, usually relieved by taking food." He was not, however, prevented attending to his work until December, 1905, though in August he consulted a medical man because of the pain after meals, and of a pain which awakened him every night from 1 to 3 a.m. In December he became worse, and in January a swelling was first noticed beneath the right costal margin which was supposed to be inflamed thell bladder tal marged gall bladder, though in January the diagnosis was again altered, as the urine was noticed to be red and to contain blood, when kidney trouble was diagnosed. In February the right side of the chest was found to be dull, evidently due to some effusion
in the pleura, and at that time the chest was aspirated, and in the pleura, and at that time the chest was aspirated, and 2 pints of clear fluid withdrawn. The diagnosis was changed to malignant tumour with secondary pleuritic effusion. The
patient was then coughing up a quantity of muco-purulent sputum, and was rapidly becoming weaker

When I saw him, on February 22nd, 1906, he was very ill, and emaciated to an extreme degree. He was suffering from irregular fever with profuse sweats, and his pulse was rapid 
and feeble. I found well-marked dullness in front as high as and feeble. I found well-marked dullness in front as high as tender mass below the right costal margin, which did not move tender mass below the right costal margin, which did not move during respiration. The right side of the abdomen was absomoved moderately. The outline of the stomach could be seen down to the level of the umbilicus, and while I was examining the abdomen there was visible gastric contraction, followed by a gurgle as if air and fluid were passing through some stricture. On auscultating the dull area on the right side of the thorax the gurgle I felt on palpating the stomach could be distinctly heard in the chest, and on percussing with two coins in the epigastrium and over the first part of the duodenum, the marked percussion note was distinctly conveyed through the stethoscope, applied over the area of dullness. With the history that I had just elicited to guide dullness. With the history that I had just elicited to guide me and with the well marked physical signs I have mentioned, I had no hesitation in diagnosing subphrenic abscess due to perforation of a duodenal ulcer, and it became clear that the abscess had burst into the lung and was giving rise to the purulent expectoration, while the pleurisy was a secondary and superadded condition.

The patient was moved to a nursing home, and on March 3rd I made an incision between the seventh and eighth ribs in the axillary line over the centre of the area of amphoric resonance. An exploring syringe was passed, and offensive pus was at once obtained. The incision was then extended into the abscess cavity, and over a pint of very fetid pus was evacuated and a large tube inserted. So far as could be made out, the pus was situated between the liver and diaphragm. He forthwith began to improve and the temperature at once became normal.

After the discharge had lessened to a certain extent, it did not tend to diminish further; and as he began to take more food the discharge increased, and with the increase of food the digestive symptoms reappeared. It was therefore decided to attack the abdominal disease, and on April 7 th, about a month attack the abdominal disease, and on April 7th, about a month after the frst operation, I opened the abdomen a little to the
right of the mid-line, and found a large ulcer of the pyloric end of the stomach extending through the pylorus into the duodenum. The pylorus and duodenum were firmly fixed to the under surface of the liver and to the front of the kidney by such firm adhesions that it was felt advisable not to disturb them, as they were doubtless bounding the abscess cavity. The cardiac end of the stomach, though dilated, seemed to be healthy, and there was no great difficulty in performing a posterior gastro-enterostomy, from which he made an uninterrupted recovery. He was able to take food by the mouth on the day of operation, and by the end of the week he was taking a large quantity of pounded fish, meats, and other foods without discomfort. The discharge from the chest mmediately began to lessen, and within a week the drainage tube was removed. Within a month from the time of the gastro-enterostomy he was able to leave the nursing home with
both wounds soundly healed, and the tumour that had been felt below the right costal margin had entirely disappeared. Welt below the right costal margin had entirely disappeared. month, and I have since heard that he is in perfect health.

Acute Pancreatitis.

One of the most fatal of abdominal inflammations is acute pancreatitis, which is characterized by symptoms pointing to acute septic peritonitis starting in the upper abdomen. It may have been preceded by symptoms of gall stones, of which pancreatitis is a frequent complication, or it may occur without apparent cause.

Be the cause what it may, as soon as diagnosed or even suspected, the abdomen should be opened by a vertical incision a little to the right of the mid-line, starting above and continuing downwards beyond the umbilicus. The focus of inflammation can then be reached, either through the great omentum below the stomach or through the lesser omentum above the stomach, or through the meso. colon by raising the transverse colon; the aim should be to incise and freely drain the inflamed area, packing of the adjoining healthy peritoneum by sterile or iodoform gauze. I have had 6 cases of acute pancreatitis under my care, and have operated on 4, of which 2 recovered.

In a case of gangrenous pancreatitis in a man aged 58 a collection of fluid was opened through the great omentum above the hepatic flexure of the colon and a slough of the pancrea was extracted, after which free drainage was established. At the same time the gall bladder and all the stones within reach were removed, but the common duct was not opened, as the patient was too ill to bear a prolongation of the operation. Fortunately several small calculi worked back through the tub in the gall bladder, and recovery was not delayed and was ultimately complete. The pancreatic reaction was well marked in this case.

In anothe: case, of a young married woman suffering from acute suppurative pancreatitis, the viscera were found hopelessly matted together. There was extensive fat necrosis all over the abdomen. A subphrenic abscess, containing masses of necrosed fat and dark pus, was evacuated. The patient was only temporarily relieved, and succumbed on the third day. In this case it would probably have been better to have drained the front, but the patient was so ill that it did not appear to be advisable to do more, lest death should occur on the table.

In another case, of a middle-aged medical man, the diffuse at necrosis and adhesions of the viscera and omentum into a dense mass presented a formidable obstacle to complete exploration; but as no evidence of any collection of fluid, either in the pancreas or in the lesser peritoneal sac, could be obtained, and as no gall stones could be felt in the gall bladder or bile ducts, the peritoneal toilet was performed and the abdomen closed, recovery following, and ending in complete restoration to health. It is worthy of note that in this case the diagnosis was confirmed before operation by the urinary pancreatic reaction.

Even if there has been a difficulty in diagnosis before the abdomen is opened, the presence of fat necrosis at once demonstrates the true condition directly the abdomen is opened. In some exceptional cases, after making the diagnosis by an abdominal incision, it may be wise also to establish drainage of the lesser peritoneal sac through the left costo-vertebral angle by means of a stab puncture from within. I performed this operation in 1888, and, although the patient succumbed to general peritonitis, which was present at the time of operation, yet the drainage of the lesser peritoneal sac had been efficiently maintained.

\section{Subacute Pancreatitis.}

Subacute pancreatitis, leading to abscess of the pancreas, is characterized by less acute symptoms, though the ultimate issue may be equally dangerous. In a case I saw recently the diagnosis was so obscure that all it was possible to affirm was that there was peritonitis starting in the upper abdomen, until the abdomen was opened, when the presence of fat necrosis associated with offensive pus clearly showed the nature of the illness.

Out of 9 cases of abscess of the pancreas that I have seen I have operated on 7, with recovery from operation in 6 , though in two cases the relief was only for a few weeks and in one for a few months.

The presence of a tumour or of a diffuse epigastric swelling behind the stomach will be generally found, or if epigastric tenderness prevents palpation, an anaesthetic will enable the swelling to be felt. There is usually fever of a septic type. The presence of leucocytosis and the discovery of the pancreatic reaction in the urine will afford valuable aids to diagnosis. As soon as an abscess forms it may reach the surface above or below the stomach, in either loin, in the left iliac region, under the diaphragm, or even in the pelvis, and will require differential diagnosis from other conditions leading to a collection of pus in those situations, such as chronic perforative gastric or duodenal ulcer, suppurative cholecystitis, splenic abscess, perirenal abscess, spinal abscess, glandular abscess, etc.

Calculus of the pancreas, though an extremely rare con dition, must not be lost sight of as a cause of inflamma. tion in the upper abdomen. I have fully reported, in a work on diseases of the pancreas, the case of a lady of advanced age in which $I$ removed several pancreatic calculi with complete success.

The diagnosis of pancreatic calculus may be assisted by the $x$ rays, for, as I pointed out in my Hunterian Lectures at the Royal College of Surgeons, while gall stones transmit the Roentgen rays, pancreatic calculi, which contain abundance of lime salts, are opaque to the rays. The presence of Cammidge's reaction in the urine and of an excess of neutral fat in the faeces, from the associated condition of interstitial pancreatitis, are also of valuable assistance.

Suppuration around Cancerous Tumours.

Suppuration around cancerous tumours of the colon, pylorus, or gall bladder, is apt to lead to great difficulties in diagnosis.

It is well known that abdominal cancer, if it does not produce obstruction or does not invade the parietal peritoneum, may be present for a considerable time before attracting attention, and that the first symptoms may only occur when the disease is complicated by the presence of infective organisms leading to inflammation or suppura. tion. Hence, the presence of an abscess in the upper abdomen at or after middle age arising without apparent cause must always be viewed with some suspicion.

I was asked some time ago to see a gentleman of 64 who was supposed to be suffering from abscess in the right hypochondrium due to cholecystitis. The abscess and inflammation in the upper abdomen were manifest, but the history of ill health 
without definite symptoms for some months and the absence of any symptoms pointing to gall stones gave rise to a suspicion of suppuration secondary to cancer, which proved to be the case, and the drainage of an offensive abscess beneath the right costal margin had to be followed by an operation for the relief of obstruction due to cancer of the hepatic flexure of the colon.

I have seen the same course of events in other cases of cancer of the colon as well as in cancer of the pylorus and of the body of the stomach and not infrequently in cancer of the gall bladder. In the latter case jaundice is usually, though not always, present. Were it desirable I could give notes of cases illustrating all these conditions in which operation has primarily been undertaken for abdominal abscess. The practical outcome of my experience is that it is better', when suppuration is combined with cancer, to do as little as possible, as not only can little good be done, but in all probability a fatal result will follow any attempt to do more than drain the abscess; or even should recovery occur after a radical operation, relapse owing to diffusion of the malignant disease will be so speedy that the slight prolongation of life is practically worthless.

\section{Jejunal Ulcer.}

This is fortunately a rare form of peptic ulcer which claims two causes, sepsis and hyperchlorhydria. In duodenal or gastric ulcers that have failed to yield to medical treatment, and in which gastro-enterostomy has been performed, I fear that the fact has not been properly grasped that the operation, though an important one, is still only an incident in the treatment, which ought to be continued on general lines for some time longer, or until good health is again established. Greater attention to oral sepsis and to the gastric condition of hyperchlorhydria subsequent to operation is advisable, and in this way the very serious complication of peptic ulcer in the jejunum, and of relapses in gastric ulcer might be prevented.

In the cases reported, perforation, associated with acute, subacute, or chronic symptoms, has occurred; hence there can be no question as to the desirability-nay, as to the absolute necessity - of operation, which ought not to be delayed too long. When the abdomen is opened the treatment will depend on the condition found. If perforation has occurred into the general peritoneal cavity, the condition will be one of the utmost peril, and only capable of relief by immediate cleansing or drainage of the peritoneum and closure of the opening, or excision of the ulcerated area.

Though peptic ulcer of the jejunum is less frequent after posterior gastro-enterostomy, and is hardly, if ever, seen when the operation is performed without a loop, yet, when it does occur, it is more likely to be acute and not to lead to adhesions, which usually occur in jejunal ulcer following anterior gastro-enterostomy.

If, as in the greater number of cases, adhesions have formed, the condition will be less acute, although very distressing from the associated pain due to perigastritis. It will be necessary to detach adhesions and to repair the perforation, but in the greater number of cases an excision of the portion of intestine involved and the performance of a Roux's operation will give the best results.

I have operated on 5 cases, and I am glad to be able to say that all recovered. In 3 I completely excised the ulcerated jejunal loop, uniting the intestine end to end, and closing the opening into the stomach, as the pyloric or duodenal ulcers, for which the gastro-enterostomy had originally been done by myself or other surgeons, appeared to have completely healed. In another I excised the ulcerated area and performed a Roux's operation.

My first case, which occurred nearly three years and a half after an anterior gastro-enterostomy performed for pyloric ulcer,
was operated on in May, 1903. The patient had marked tenderwess in the epigastrium, associated with a definite swelling and wess in the epigastrium, associated with a definite swelling and with rigidity of both recti. For some time previously he had
been subject to severe attacks of epigastric pain, and blood had been subject to severe attacks of epigastric pain, and blood bac

been noticed in the stools, but there had been no vomiting. The abdomen was opened by an incision just to the right of
the middle line, and the stomach was found adherent to the anterior abdominal wall, 1 in. to the left of the old scar.

On separating the adhesions it was found that ulceration had occurred at the point of junction between the bowel and the stomach, and that it had involved the whole circumference of the jejunum at that point, the ulceration having completely extended through the intestinal wall at the front; but this must have occurred after adhesions, so that there had been no acute symptoms of perforation. Through the opening left when the adhesions had been separated the finger was passed into the stomach, when it was found that the pylorus had quite closed as a result of the ulceration threa years before, so that nothing could have passed through it. On passing the finger into the intestine extensive ulceration and thickening were discovered. The ulcerated portion of the bowel was completely excised and then separated from the opening in the stomach, the edges of which were pared, the distal end of the jejunal loop. was implanted into the old opening in the stomach, and the proximal patient made a steady recovery, and returned home within the month.

Liver.

Either liver abscess or suppurating hydatid disease may lead to inflammatory trouble in the upper abdomen, necessitating surgical interference, though the previous history and the presence of a tumour or of enlargement of the liver will usually render the diagnosis sufficiently evident, even before the abdomen is opened or before pus is discovered by the exploring syringe.

Did time allow, I could relate a number of cases illustrating the condition.

\section{Retroperitoneal Glands.}

Suppurating retroperitoneal glands forming a tender swelling above the umbilicus have on two occasions led me to fear more serious conditions, such as tumour of the posterior wall of the stomach or of the pancreas. In both cases superficial resonance on percussion and dullness on deep pressure showed that the swelling was behind the stomach, and in both I was able successfully either to excise the inflamed glands or to clean out the abscess connected with them.

\section{Other Conditions.}

Of other conditions that I have seen to produce inflam. mation in the upper abdomen I have only time to brietly mention: (1) A perforated colic pouch setting up peritonitis limited by omental adhesions, and which was cured by excising the portion of colon involved; (2) ruptured cancerous ulcers of the colon and of the stomach; (3) splenic abscess; (4) subdiaphragmatic abscess; (5) volvulus of the stomach ; (6) phlegmonous gastritis; and (7) acute dilatation of the stomach.

On the latter condition I must make a few remarks, as I have seen a number of these alarming cases, the treatment of which, if recognized sufficiently early, may be successful, whereas, if undiagnosed, the condition is rapidly fatal.

It may occur without apparent cause or subsequent to. any abdominal operation, and is characterized by the sudden onset of severe epigastric pain with shock, incessant. but ineffectual vomiting, suppression of urine, great abdominal distress, breathlessness, rapid pulse, lowered tem perature, and enormous distension of the abdomen, at first of the upper part and later of the abdomen generally. The face becomes pinched and sunken, and the expression is extremely anxious. On percussion the stomach reson. ance reaches well up into the thorax, displacing the heart and limiting chest expansion.

In the great majority of cases the issue has been fatal, and all treatment has been unavailing. But the disease is by no means necessarily lethal, and I have, in my own experience, three cases to record in which the symptoms have subsided, and the patients progressed to recovery.

In the early stages recourse should be had at once to lavage of the stomach. If the organ rapidly fills after it is emptied, the tube may be left in for several hours. The position of the patient must be altered, so that he lies prone in bed, with a pillow under the pelvis and the lower part of the abdomen. In the severer instances, it may be necessary to open the stomach and drain, performing, in fact, a gastrostomy; or, as I suggested some time ago, recourse may be had to gastro.enterostomy, whereby the stomach is drained continuously into the intestines. The latter operation has, I believe, only once been put to the proof, and that in a case of my own, which was followed by recovery; but on theoretical grounds, and from $\mathrm{my}$ experience in other diseases of the stomach, it would seem likely to be of service. The fact that gastrostomy has always proved fatal need not deter the surgeon, if other means have failed, for an examination of the recorded cases shows that all patients upon whom it was practised were moribund or almost in the last extremity. 
But that, the condition of acute gastric dilatation, when recognized early, is capable of being successfully treated, the following two cases distinctly prove:

In one, a woman of 35, recovering smoothly from cholecystotomy, which had been performed a week previously, was suddenly seized with pain in the epigastrium, followed by vomiting, which soon became ineffectual in emptying the stomach. Rapid dilatation ensued, and the stomach not only formed a large swelling, filling up the superior abdominal region, but also extended well below the umbilicus towards the pubes. This, owing to pressure on the diaphragm, and through it to pressure on the heart and lungs, led to great shock, quick and oppressed breathing, and a rapid, feeble pulse, with qigns and oppressed breathing, and a rapid, feeble pulse, with sign of lividity and imperfect blood aëration. The urine wa scanty, and in fact almnst suppressed: Strychnine was freely given subcutaneously, but no relief came until the stomach tube was used, when a large quantity of gas and several pints of brownish fluid were evacuated; the lavage was repeated several times during the next two days, during which time alimentation was entirely rectal. It was interesting to note that the pulse and temperature were paradoxical, the former running up to 150 and the latter down to $97.3^{\circ}$. All the symptoms passed off under treatment almost as quickly as they came, and in a week the stomach had returned to the norma size.

In another case, a patient of 29 , who had had abdominal hysterectomy for a large myoma, and whose after-progress had been most satisfactory, the wound having healed by first intention, and the temperature and pulse having been normal throughout, was seized with pain over the stomsch a fortnight after operation; this was followed by vomiting of frothy mucus with a few brownish streaks in it, and by distension of the with a few brownish streaks in it, and by distension of the abdomen beginning in the epigastrium. Cn percussion, the stomach was found to be down to the pubes, and on succussion a well-marked splash was easily obtained; the pulse becamo rapid and the face pinched, so that within thirty-six hours of impending death. The urine was very much diminished, and for twenty-four hours almost suppressed. Strychnine was given subcutaneously, and rectal feeding was adopted, but no relief was. obtained until the stomach was washed out after which relief was immediate and the patient steadily improved, thoug no fod wo though hood a week the stomach had returned to the normal size. Here also the temperature and pulse were paradoxical, the former bein subnormal and the latter very rapid. The only cause that could be assigned was the eating of a raw apple, but that may have had nothing to do with the condition. The patient said that she had always been subject to a weak digestion since childhood, but at the time of operation nothing abnormal was
noticed in the stomach.

I feel, Sir, that I ought to apologize for taking up a subject that almost necessarily from its magnitude has had to be treated in a discursive manner, and in a way that makes it merely suggestive rather than complete But if my address should have the practical value of showing the importance of an attempt to make an early diagnosis with a view to speedy treatment in a class of cases which, if left undiagnosed, are prone to end fatally with great rapidity, I shall feel that your kind indulgence has not been imposed on.

\section{A Cliniral Ierture}

oN

\section{SOME OF THE RARER ASSOCIATIONS}

OF GALL STONES AND BILIARY OBSTRUCTION.

BY

T. CA R WARDINE, M.S.Lond, F.R C.S., SCI:GioN TO THE BRISTOL ROYAL INFIRMARY.

The presence of several gall bladder cases in my female ward recently is an indication of a suitable subject to bring before you, but I will eliminate the consideration of ordinary cases; an adequate description of which can be found in modern surgical textbooks, and limit my remarks to some of the rarer associations, some of which you have had the opportunity of seeing.

\section{A Congenital Peritoneal Fold as a Cause of Chronic Cholecystitis.}

It is not uncommon to find the neck of the gall bladder connected with the lower margin of the foramen of Winslow by a fold of peritoneum, which is usually small. This was observed by Greig Smith, ${ }^{1}$ and Dr. G. E. Brewer ${ }^{2}$ noticed it in 3 per cent. of cases. In a patient recently under my care this fold extended the full length of the gall bladder, reaching the top of the fundus, binding the gall bladder down in such a way that the cystic duct was kinked ; its opening into the gall bladder was eccentrically placed (Fig. 1). The lady was three months pregnant, and had discovered a hard, globular lump about a year previously, with little tenderness and no symptoms referable to it. The fold was freely divided, and the gall bladder, containing thick, tarry bile, was drained, with complete relief to the condition. There seems to be an occasional tendency for the gall bladder to be enclosed in the free border of the gastro-hepatic omentum, which explains the anomaly referred to. In one dissec. tion Brewer found the gall

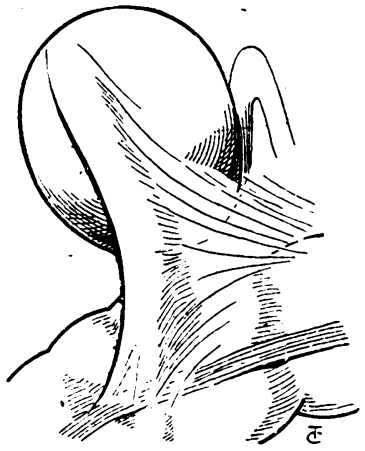

Fig. 1.-Congenital peritoneal fold. bladder between the two folds of the gastro-hepatic omentum, the free border of which extended well to the right, from the anterior margin of the right lobe of the liver to the hepatic flexure of the colon.

General Peritonitis Originating from the Gall Bladder.

In the slighter degrees of inflammation of the gall bladder from contained stones and infection a little fluid is found around, and translucent, soft gelatinous lymph in contiguity, agglutinating the surrounding parts in the same manner as obtains in infective appendicitis. This may clear up; but in the more severe cases the lymph organizes into firm fibrous tissue, binding the parts firmly together by strong adhesions, which may also pervade the surface of the liver. The adhesions are conspicuous in old-standing cases, especially those with small, contracted gall bladders. In many instances the degree of local peritonitis is greater, although with rest and treat. ment it usually diminishes, when the surgeon may select a more favourable opportunity for cholecystostomy. General peritonitis without perforation is a rarer event.

A woman, aged 68, who bad hemiplegia a year previously, was admitted with general peritonitis and vomiting, suggesting intestinal obstruction as a probable cause. The acute illness began five days previously with sudden abdominal pain and sickness. On making a subumbilical incision much fluid and sickness. On making a subumbilical incision much fluid and lymph escaped, the appendix and pelvic organs were normal, some filamentous peritoneal adhesions were met with, and there was no evidence of intestinal obstruction. The hand was then
passed to the right bypochondrium, whence much fluid escaped, and a large, tense gall bladder was felt containing stones. Three large faceted calculi were removed, the gall bladder and right hypochondrium were drained, and two very large drainage tubes were put into the pelvis. The patient made a good recovery.

I have dealt with the advantage of peritoneal drainage elsewhere, ${ }^{8}$ which I freely practise and strongly advocate in the treatment of general peritonitis.

Alternating Cholecystitis and Biliary Colic.

In the surgery of the gall bladder and common bile duct, the "ball-valve" action of floating stones is now well recognized, temporarily occluding the cystic duct on the one hand or the lower part of the common duct on the other hand. Fenger first drew attention to this mechanical occurrence in the common duct. ${ }^{4}$ When the gall bladder contains a large ovoid stone, as well as many small and irregular ones, it is conceivable that the large stone may act as a trap, which, when opened, will permit a discharge of fluid and small stones; and the latter, in their turn, lodging in the common duct, will give rise to attacks of biliary colic with jaundice. The distended gall bladder would contract at the time of the flux caused by the open. ing of the ball-valve. This is the obvious explanation of a case first seen with Dr. Flemming and since operated upon (Fig. 2, $A$ and $B$ ).

The patient, a man aged 55, gave a history of two attacks of "indigestion"; and vomiting without jaundice, but with enlargement of the gall bladder, during the previous year. At the end of the year he had several slight attacks, followed by a severe of the year he had several slight attacks, followed by a severe urine and jaundice for the first time, but without enlargement of 